We thank the Bacteriology Department for performing the urine cultures and $T$ Dealy for secretarial assistance.

\author{
References \\ 1 Källenius G, Winberg J. Urinary tract infections treated \\ with single dose of short-acting sulphonamide. $\mathrm{Br} \mathrm{Med} J$ \\ 1979 ; i: 1175-6. \\ 2 Bailey R R, Abbott G D. Treatment of urinary tract \\ infection with a single dose of trimethoprim-sulfa- \\ methoxazole. Can Med Assoc J 1978; 118: 551-2. \\ 3 Fairley K F, Whitworth J A, Kincaid-Smith P, Durman O. \\ Single dose therapy in management of urinary tract
} infection. Med J Aust 1978; ii : 75-6.
4 Bailey $R$ R, Abbott G D. Treatment of urinary-tract infection with a single dose of amoxycillin. Nephron 1977; 18: 316-20.

5 Russ G R, Mathew T H, Caon A. Single day or single dose treatment of urinary tract infection with cotrimoxazole. Aust NZ J Med 1980; 10: 604-7.

- Rubin R H, Swartz M N. Trimethoprim-sulfamethoxazole. $N$ Engl J Med 1980 ; 303 : 426-32.

7 Lipson A, Marshall W C, Hayward A R. Treatment of Pneumocystis carinii pneumonia in children. Arch Dis Child 1977; 52: 314-9.

Correspondence to Dr J R Burke, Mater Children's Hospital, South Brisbane, Queensland 4101, Australia.

Received 27 October 1981

\title{
Timing of neonatal cerebroventricular haemorrhage with ultrasound
}

\author{
L CH DE CRESPIGNY, R MACKAY, L J MURTON, R N D ROY, AND P H ROBINSON \\ Department of Obstetrics and Gynaecology, University of Melbourne, Royal Women's Hospital, and \\ Division of Paediatrics, Royal Women's Hospital, Melbourne, Australia
}

SUMMARY Sequential real-time ultrasound examinations were performed in 174 neonates to determine the time of occurrence of cerebroventricular haemorrhage (CVH). Of the 47 infants in whom CVH was detected, in $36(77 \%) \mathrm{CVH}$ was present at the first examination. Of the 34 infants with CVH who were examined first within 6 hours of birth, $24(71 \%)$ already had haemorrhage demonstrable at the initial scan. Extension of a CVH after its initial detection occurred in only 3 infants. Of 124 consecutive infants of birthweights less than $1500 \mathrm{~g}, 38(31 \%)$ developed CVH, $56 \%$ of the outborn and $27 \%$ of the inborn babies. Our results indicate that most infants who develop CVH have done so within 6 hours of birth.

Real-time ultrasound equipment has assumed an important role in the detection of intraventricular haemorrhage in preterm infants. Since there is no known risk of the procedure and minimal disturbance to the neonate during its use, we considered it could be employed serially to time the occurrence of cerebroventricular haemorrhages (CVH) (that is germinal layer or intraventricular haemorrhage).

\section{Subjects and methods}

During a 12-month period all infants weighing less than $1500 \mathrm{~g}$ at birth, together with those exceeding this weight who had other risk factors-such as severe hyaline membrane disease requiring assisted ventilation-were examined using an ADR real-time ultrasound scanner with a $7 \mathrm{MHz}$ linear array transducer. Examinations were performed as soon as possible after birth, repeated daily for 3 days, and then again at one week. The brain was examined using a series of oblique coronal sections through the anterior fontanelle by angling the transducer forwards then slowly rotating it backwards with the anterior fontanelle as the fulcrum. CVH was diagnosed when the appearance of echogenic blood clot was visible in, or immediately inferolateral to, the lateral ventricle. The extent of the haemorrhage in the anteroposterior direction was assessed by an oblique parasagittal scan. We had confirmed the accuracy of our technique previously by comparison with computerised tomography (CT) scans and necropsy results. ${ }^{1}$

\section{Results}

A total of 174 infants was studied. In $47 \mathrm{CVH}$ was detected. Of the 124 infants of birthweight less than $1500 \mathrm{~g}, 38(31 \%)$ developed CVH. Of the 16 infants of birthweight less than $1500 \mathrm{~g}$ who were outborn, $9(56 \%)$ developed CVH (all these being present at the time of admission to our hospital), while haemorrhage occurred in $29(27 \%)$ of the 108 very low birthweight infants born within the hospital. This difference was statistically significant $(P=0.04$, Fisher's exact test).

Haemorrhages in infants of birthweight less than $1500 \mathrm{~g}$ were classified as follows: 13 haemorrhages 
were grade I (less than $1 \mathrm{~cm}$ in size, presumed germinal layer), 10 grade II (filling less than half the lateral ventricle), 9 grade III (filling more than half the lateral ventricle), and 6 grade IV (extending into the brain parenchyma). In the infants of birthweight greater than $1500 \mathrm{~g}, 2,4,2$, and 1 were grade I, II, III, and IV respectively. Examination at necropsy was performed in 10 of the 15 infants who died and in each the ultrasound findings were confirmed.

Ultrasound examination was performed within 6 hours of birth in 34 infants with CVH and in 24 ( $71 \%$ ) of them signs of the haemorrhage were already present. Furthermore, of the 47 infants who had a $\mathrm{CVH}$, the ultrasound features were already present in 36 at the time of the first examination (Table 1). In the remaining 11 babies no haemorrhage was detected at the time of the first examination, but was visualised in the first 48 hours in 9 , while haemorrhage occurred on day 3 in 2 infants. In 6 of the 11 infants who were noted as developing $\mathrm{CVH}$ after a normal scan, severe respiratory complications occurred at the time of the haemorrhage: blocked endotracheal tube $(n=2)$, pneumothorax $(n=1)$, or severe apnoea $(n=3)$. Ultrasound evidence of extension of the CVH after its initial detection occurred in 3 of the 47 infants. The high incidence of early haemorrhage in this study was not owing to large numbers of either extremely preterm or small-for-gestational-age babies (Table 2).

Table 1 Time of detection of cerebroventricular haemorrhage

\begin{tabular}{ll}
\hline $\begin{array}{l}\text { Time of examination } \\
\text { (hours after birth) }\end{array}$ & Number of infants \\
\hline Detected at first examination $(\mathrm{n}=36)$ & \\
1 hour & 10 \\
$1-<2$ & 4 \\
$2-<3$ & 3 \\
$3-<4$ & 4 \\
$4-<6$ & 3 \\
$6-<24$ & 8 \\
$24-36$ & 4 \\
& \\
Detected after first examination $(n=11)$ & 9 \\
$1-<48$ & 2 \\
$48-72$ & \\
\hline
\end{tabular}

Table 2 Birthweight and gestational age of infants with cerebroventricular haemorrhage

\begin{tabular}{lll}
\hline $\begin{array}{l}\text { Birthweight } \\
(g)\end{array}$ & $\begin{array}{l}\text { Total no of infants } \\
(n=47)\end{array}$ & $\begin{array}{l}\text { No of SGA infants } \\
(n=4)\end{array}$ \\
\hline $500-750$ & 2 & 0 \\
$751-1000$ & 7 & 2 \\
$1001-1250$ & 14 & 1 \\
$1251-1500$ & 15 & 1 \\
$>1501$ & 9 & 0 \\
\hline
\end{tabular}

SGA $=$ small-for-gestational age (weight less than 10th centile).

\section{Discussion}

We have shown that most $\mathrm{CVH}$ s are already present within 6 hours of birth and that rarely they occur after 48 hours. In this study only 11 (23\%) of the 47 infants developed CVH after the first ultrasound examination. Once CVH was detected, extension of the lesion was found to be rare ( 3 of 47 babies). The incidence of $\mathrm{CVH}$ was $31 \%$ in our series of infants less than $1500 \mathrm{~g}$ birthweight, with a low incidence in inborn infants $(27 \%)$, while in outborn infants the incidence of $56 \%$ was similar to that found by others. This difference may be due to bias in selection of neonates admitted to this hospital or to problems of neonatal transport, but it may well reflect differences in the quality of perinatal care. Although most haemorrhages were present early in neonatal life and were not associated with pneumothorax, ${ }^{2}$ pulmonary complications occurred at the time of $\mathrm{CVH}$ in 6 of the 11 infants documented as developing haemorrhage after birth.

Studies at necropsy suggest that CVH occurs early in the neonatal period and is rare before birth. ${ }^{3}$ Using indirect methods of clot analysis, it has been estimated that most CVHs occur after the first 24 hours of life, ${ }^{4}$ or between 10 and 36 hours. ${ }^{5}$ Lee et al. ${ }^{6}$ attempted to time the occurrence of $\mathrm{CVH}$ using CT scans and showed that a haemorrhage was already present in 9 of 11 babies who were examined within the first 24 hours of life. Such findings are more in agreement with those of this study. Using ultrasound, Bejar et al. ${ }^{7}$ also found that most haemorrhages were present from close to the time of birth, but their extremely high incidence of $\mathrm{CVH}$ is out of keeping with others. Our results contrast with a recent study by Levene et $_{\text {al }}{ }^{8}$ who found that most haemorrhages occurred after the first day of life. In our study, which included a greater number of neonates, scans were performed closer to the time of birth.

Our findings have implications regarding the management of very low birthweight infants. If, as our evidence suggests, CVH occurs either before delivery or very early in the neonatal period, the main aetiological factors are likely to be operative at this time and later complications-such as tension pneumothorax-are less frequent although potent, precipitating factors. From our continuing studies we hope to determine if $\mathrm{CVH}$ precedes delivery or is a condition that is solely related to neonatal factors. In this way a better understanding of the aetiology of the condition may emerge, thus allowing identification of preventable factors.

We thank the medical and nursing staff of the Paediatric Department for assistance, and Professor Roger Pepperell for advice and encouragement. 
ADR Ultrasound (Tempe, Arizona) supplied the ultrasound equipment.

\footnotetext{
References

1 de Crespigny L C, Robinson $\mathbf{H}$ P, Murton L J, O'Herlihy C. Real-time scanning of the neonatal brain. Aust Paediatr J 1981 ; 17 : 78-82.

2 Lipscomb A P, Reynolds E O R, Blackwell R J, et al. Pneumothorax and cerebral haemorrhage in preterm infants. Lancet 1981 ; i: 414-6.

3 Leech R W, Kohnen P. Subependymal and intraventricular hemorrhages in the newborn. Am J Pathol 1974; 77: 465-75.

4 Tsiantos A, Victorin L, Relier J P, et al. Intracranial hemorrhage in the prematurely born infant: timing of clots and evaluation of clinical signs and symptoms. J Pediatr 1974; 85: 854-9.

5 Emerson $\mathbf{P}$, Fujimua $\mathrm{M}$, Howat $\mathrm{P}$, et al. Timing of intraventricular haemorrhage. Arch Dis Child 1977; 52: 183-7.
}

6 Lee B C P, Grassi A E, Schechner S, Auld P A M. Neonatal intraventricular hemorrhage: a serial computed tomography study. J Comput Assist Tomogr 1979; 3: 483-90.

7 Bejar R, Curbelo V, Coen R W, Leopold G, James H, Gluck L. Diagnosis and follow-up of intraventricular and intracerebral hemorrhages by ultrasound studies of infant's brain through the fontanelles and sutures. Pediatrics 1980; 66: 661-73.

8 Levene M I, Wigglesworth J S, Dubowitz V. Cerebral structure and intraventricular haemorrhage in the neonate: a real-time ultrasound study. Arch Dis Child 1981 ; 56: 416-24.

Correspondence to Dr L Ch de Crespigny, University of Melbourne, Department of Obstetrics and Gynaecology, Parkville, Victoria 3052, Australia.

Received 9 October 1981

\title{
Normal values for mature and immature neutrophils in very preterm babies
}

\author{
B W LLOYD AND A OTO \\ Department of Paediatrics and Neonatal Medicine, Hammersmith Hospital, London
}

SUMMARY Serial leucocyte counts were made on 24 babies of less than 33 weeks' gestation during the first 5 days of life. The values for mature neutrophils show two important differences from those obtained by previous workers studying term babies, in that the counts were much lower on the first day of life and showed a wider range of values at all ages. In contrast, immature neutrophil counts, expressed either as absolute values or as a proportion of the total neutrophil count (the I/T ratio), were similar to those found in previous studies and had a considerably narrower range.

Neutrophil counts are widely used to help diagnose infection in newborn babies. ${ }^{1-4}$ Published reference ranges have been largely constructed using data from term or fairly mature preterm babies. ${ }^{2} 56$ It has been suggested ${ }^{1257}$ that the neutrophil counts of preterm babies differ from those of term babies, casting doubt on the usefulness of these ranges when dealing with the very immature. Previous work on preterm babies has been with fairly mature infants ${ }^{15}$ (M Xanthou and E Hey, 1981, personal communications), so we set out to determine the range of mature and immature neutrophil counts in healthy babies of not more than 32 weeks' gestation.

\section{Patients and methods}

Twenty-four babies admitted to the neonatal intensive care unit at Hammersmith Hospital between June 1979 and August 1980 were studied with their parents' written consent and the approval of the hospital's ethical committee. All were 32 weeks' gestation or less according to their mothers' dates, and gestational assessment by the method of Dubowitz et al. ${ }^{8}$ Mean gestational age was 30.4 weeks (range 26-32) and mean birthweight $1510 \mathrm{~g}$ (range 900-1900).

Certain conditions were fulfilled to ensure that the infants could reasonably be considered healthy. There were 3 babies born after prolonged rupture of membranes and 8 with very mild and short-lived respiratory distress who had no clinical or laboratory evidence of infection.

Blood was taken from each baby at 1, 12, $24,48,72,96$, and 120 hours, although specimens were not obtained at every age from all. A total of 124 specimens was taken from 24 babies using capillary blood from the heel; $0.2 \mathrm{ml}$ of blood was taken into a tube containing $0.3 \mathrm{mg}$ of dipotassium EDTA. A total leucocyte count was performed using a Coulter counter. A blood film was stained using a standard May Grunwald (Giemsa) 